\title{
LES PERSPECTIVES DE RELANCE DU DEVELOPPEMENT AGRICOLE ET DE L'AUTOSUFFISANCE ALIMENTAIRE DE LA RDC SUITE A L'ADOPTION D'UN CODE AGRICOLE EN MAI 2011
}

\author{
L'exemple de la province de Katanga
}

\section{Par Baudouin WIKHA TSHIBINDA et Robert NKUMISONGO*}

\section{INTRODUCTION}

La République Démocratique du Congo est un pays à vocation agro-pastorale. Près de $70 \%$ de la population vit en milieu rural et dépend essentiellement de l'agriculture. Le potentiel que ce secteur offre devait permettre la RD. Congo de jouer un rôle important pour son développement socioéconomique.

Les possibilités d'une relance agricole en République Démocratique du Congo (RDC), ainsi que les problèmes à adresser, sont multiples.

Cette étude vise à présenter de quelques aspects agronomiques nécessaires pour un développement durable, aussi bien sur le plan socio-économique, que sur le plan technique de la province du Katanga après l'adoption du code agricole de notre pays.

De manière paradoxale, le pays enregistre, depuis plusieurs années, une forte régression en matière agricole. Il se trouve classer parmi les pays pauvres de la planète. La production intérieure n'est plus en mesure de répondre à la demande alimentaire.

Le code agricole couvre l'ensemble des activités économiques du secteur agricole et péri agricole; il s'agit de l'agriculture, l'élevage, la pêche et la pisciculture, l'aquaculture, l'apiculture, la chasse, l'agro foresterie, la transformation, le transport, le commerce de produits agricoles et l'agroalimentaire, la distribution et d'autres services agricoles, ainsi que leurs fonctions sociales et environnementales.

Le but principal est de créer de meilleures conditions socio-économiques pour le monde rural. Ceci demande la promotion aussi bien des cultures vivrières que des cultures de rente. En même temps, pour augmenter les revenus des cultivateurs, il faut promouvoir une agroindustrie assurant la transformation après récolte, et développer des systèmes de commercialisation appropriés. Une productivité accrue et un meilleur revenu des cultivateurs résulteront automatiquement dans une amélioration de l'économie nationale.

L'ambition du code est de passer d'un statut d'une agriculture de subsistance vers une intensification de la production et un statut de petit entreprenariat. Aussi d'appliquer un ar-

\footnotetext{
* Chefs de Travaux à l'Université de LUBUMBASHI.
} 
bitrage efficace de proximité sur la diversité des pratiques coutumières sur le secteur, de simplifier l'accès sécurisé à la terre au profit du paysannat et des investisseurs dans le respect de la loi foncière.

Le projet de loi portant code agricole précise qu'il est incontestable que le développement de l'agriculture est le moteur de la relance économique de la RDC et la pierre angulaire de la stratégie de lutte contre la pauvreté.

En parcourant le code agricole il ressort que l'accent économique est mis sur l'initiative locale et la promotion du développement rural.

L'économie nationale doit être entendue comme l'ensemble des activités économiques de la nation qui concourent à la production, à la distribution et à la consommation de tous les biens et services nécessaires à la satisfaction des besoins de l'ensemble de la population. ${ }^{1}$ Le code agricole congolais s'est fixé comme objectif à court terme et long terme la réduction de la pauvreté et le développement. Le grand défaut de la République Démocratique du Congo est le manque de mesures d'accompagnement des textes légaux. La politique agricole de la République est fondée sur la sécurité alimentaire et la réduction de la pauvreté. Cette politique est contenue dans les Objectifs du Millénaire pour le Développement (OMD).

Le secteur agricole est un moteur pour la croissance économique et le développement durable. La preuve est qu'il y a des pays africains qui ont atteint une stabilité économiques grâce à l'agriculture. Les efforts dans ce domaine doivent être ceux d'amener l'exploitation ménagère agricole d'un hectare et demi par an à quatre ou cinq hectares avec l'utilisation des intrants et l'utilisation du matériel moderne ou la traction animale.

Ainsi le code agricole peut-il favoriser la relance agricole et le développement de la province du Katanga?

Il est incontestable que le développement de l'agriculture est le moteur de relance économique et vu sous cet angle, il apparait nécessaire d'observer les textes légaux régissant le secteur agricole.

Mais il se constate que la population du Katanga se caractérise par une apathie face au travail de la terre. La raison est due à la vocation minière de la province et à la dégradation des infrastructures. Toute la population est concentrée dans les grands centres où les produits de première nécessité viennent des pays du sud notamment la Zambie, le Zimbabwe et l'Afrique du Sud. Les jeunes dans les villes du Katanga se sont désintéressés des travaux agricoles pour l'exploitation minière artisanale. Le boom minier dans la province du Katanga a contribué à l'abandon du secteur agricole.

Le champ de notre réflexion couvre la province du Katanga et les potentialités que regorge cette province pour le développement de l'agriculture. L'étude menée sur le Katanga après les mines, montre les potentialités que la province regorge pour le développement du secteur agricole. La politique du gouvernement provincial du Katanga consiste à demander

1 Jean Tournier, Les bases économiques et humaines de l'activité agricole, $3^{\mathrm{e}}$ édition, Paris, Lavoisier, 1989, p. 31. 
aux opérateurs miniers à coupler l'exploitation minière avec l'agriculture. Cette politique n'est pas en faveur des paysans qui représentent $70 \%$ de la population. Hors il suffit d'un bon encadrement du paysannat en lui trouvant un marché et canaliser sa production vers les centres de consommation. Pour survivre l'homme doit satisfaire des besoins élémentaires tels que se nourrir, se vêtir, se faire soigner et s'abriter. Mais exister pour l'homme, c'est aussi la recherche constante de l'amélioration de conditions de vie. ${ }^{2}$

Hormis l'introduction et la conclusion, notre réflexion portera sur deux points essentiels. La première porte sur le développement agricole pour l'autosuffisance alimentaire du Katanga et Le deuxième point analyse le code agricole et les potentialités que le code agricole offre pour le développement de l'agriculture.

\section{Du développement agricole pour l'autosuffisance alimentaire du Katanga}

1. Notions sur le développement agricole

Le discours théorique sur le développement doit pouvoir relever de la raison pratique et non seulement de la raison technicienne. Outre la science et la technique, il y a la décision individuelle et collective. Celle-ci se fonde sur des principes et sur des normes de moralité. ${ }^{3}$

Muyer 0yong dans son ouvrage « impératif du développement et la réforme de l'administration locale au Zaïre » parle de la nécessité du changement en vue du développement. Il s'agit de la création des conditions nouvelles qui trahissent l'incapacité dans laquelle se trouvent les administrations locales à y faire face et permettent de mieux comprendre les problèmes de gestion qui se posent à l'échelon local. ${ }^{4}$ Pour un pays où $70 \%$ de la population dépend essentiellement de l'agriculture, le secteur agricole doit constituer un cadre de développement économique et social qui aujourd'hui est une trame des obstacles à surmonter. L'investissement en agriculture peut être direct ou indirect. Il est direct, lorsqu'il est fait en nature (renouvellement des troupeaux); Il est indirect lorsqu'il est fait sous la forme de la monnaie.

Pour atteindre le développement, il est nécessaire de créer un cadre institutionnel qui permettra à la population d'accéder au système économique et social. La modernisation de la structure sociale, économique, idéologique et politique exige une restructuration des rapports entre les gouvernants et les peuples pour l'appropriation de la politique nationale agricole en vue d'affecter leur vie.

2 Jean Tournier, Op.cit, p. 3.

3 Okolo Okonda W'oleko, Pour une philosophie de la culture et du développement, Kinshasa, P.U.Z., p. 106.

4 Muyer Oyong, Impératif du développement et réforme de l'administration locale au Zaïre, Kinshasa, P.U.Z., 1986 p. 60. 


\section{A. Développement agricole}

Le développement agricole est un concept qui s'est substitué à la vulgarisation agricole qui avait pour objet la formation permanente technique et économique des agriculteurs dans le but d'élever la productivité des exploitations et le niveau de vie des exploitants. ${ }^{5}$ L'impératif du développement agricole nécessite une modernisation à l'échelon local qui doit passer par la connaissance de ces problèmes pour orienter les programmes de réforme dont les résultats soient satisfaisants. ${ }^{6}$

La réalisation du développement agricole ne peut se réaliser que grâce à des actions collectives qui ont pour objectif :

- La diffusion parmi les agriculteurs des connaissances nouvelles sur les techniques de productions, la gestion des exploitations, les problèmes économiques, etc.

- La prise de conscience des exploitants familiaux et des organisations professionnelles des problèmes généraux de leur profession dont les solutions intéressent leur avenir.

- Les associer à la recherche agricole appliquée par la réalisation d'études et d'expérimentation;

Les exploitations agricoles dans les milieux ruraux se sont axées sur les structures familiales, la production de la nourriture à consommer localement. Or les paysans ont besoin des ressources financières pour acheter des semences, des outils, des engrais. Ils ont aussi besoin des routes et moyens de transport pour évacuer leurs produits vers les grands centres de consommation.

Plusieurs écrits, conférences et forums ne cessent de confirmer que l'agriculture constitue la priorité absolue du développement d'un pays. Mais la situation actuelle de la République Démocratique du Congo en ce domaine n'incite pas à l'optimisme : dans le secteur agricole où plus de la moitié de la population ne vit que de la paysannerie, la pauvreté est criante.

Il nous faut donc nous rendre à l'évidence, que les stratégies suivies jusqu'à présent n'ont pas produit l'effet escompté

Pour le Katanga, la production agricole est caractérisée par trois systèmes de production :

1. Le système traditionnel ou itinérant sur le brulis. Le paysan recourt à la main d'œuvre familiale sans intrants agricoles ou avec intrants, sans un encadrement par les agronomes. Ils pratiquent une agriculture de routine.

2. le système intermédiaire dans lequel les producteurs sont organisés en une structure ou association. Le producteur accède à l'encadrement et aux infrastructures d'appui à la production. C'est dans ce cadre qu'il faut situer le recours à la traction bovine observé dans certaines contrées de la province. Sur ces cites, on observe l'accroissement des su-

5 Okolo Okonda W'oleko, Op.cit, p. 358.

6 Muyer Oyong, Op.cit, p. 60. 
perficies emblavées, la réduction du temps de travail et une certaine amélioration du mode de transport.

3. le système moderne qui exploite de grandes superficies, recourt à la mécanisation, à un usage à grande échelle des intrants agricoles et à une main d'œuvre salariée. Les pratiques agricoles qui font usage des tracteurs ont été tentées vers les années soixante dix et quatre vingt à travers certains projets du Ministère de l'Agriculture.

\section{B. Autosuffisance alimentaire}

Le concept d'autosuffisance est devenu dominant dans les politiques agricoles mises en œuvre en Afrique au milieu des années 1970. ${ }^{7}$ L'autosuffisance est devenue un devoir pour les pays africains en vue de satisfaire aux besoins fondamentaux de la population. Pour être à même d'atteindre un tel objectif, le pays devra se doter d'une politique alimentaire conséquente et dont la mise en œuvre sera matérialisée à travers une stratégie nationale de sécurité alimentaire pour l'amélioration de la qualité de vie. L'évaluation de l'autosuffisance alimentaire passe par trois indices essentiels pour montrer si un État peut prétendre à la sécurité alimentaire de sa population. Il s'agit : de l'accessibilité à la nourriture, la disponibilité de la nourriture et l'utilisation de la nourriture.

\section{Mesures agricoles}

Le développement d'un pays passe par l'électricité. A titre illustratif, les mesures sont prises pour interdire le déboisement alors que cette population n'a pas l'électricité pour acheter un réchaud, un congélateur, il continuera à couper les bois pour le chauffage et le fumoir.

Les mesures de relance du développement agricole et d'autosuffisance alimentaire doivent être envisagées dans une perspective à court et long terme.

A court terme, le code agricole au regard des « Objectifs du Millénaire pour le Développement (OMD) » s'est fixé au premier rang la réduction de moitié, à l'horizon 2015, du nombre de sous-alimentés et de celui des plus pauvres en général face à une situation préoccupante. L'indice de la pauvreté étant estimée à $70 \%$, elle est plus prononcée dans les zones rurales où $80 \%$ de la population vit avec moins d'un dollar par jour. L'insécurité alimentaire et la sous-alimentation affectent plus de $70 \%$ de la population fragilisée aussi par le paludisme et VIH/SIDA.

A long terme, l'État désire lever les options et formuler un projet de développement pour les 30 années, soit en 2041et grâce à un cadre de référence obligeant l'ensemble des acteurs agricoles, mettant en place les conditions incitatives et l'encadrement de la jeunesse à l'exploitation agricole. Le code jette les bases du financement de l'économie agricole et des infrastructures (économie rurale, encadrement du paysan, sécurité et équité dans la ré- 
partition des terres). Le code agricole offre un cadre aux entités décentralisées dans le sens de la participation active au développement régional en fonction des avantages comparatifs de chaque province.

La meilleure politique serait de cibler les paysans, d'inventorier les demandes en matière de sécurité alimentaire, d'aménager le circuit d'évacuation, d'affecter des agronomes dans les milieux paysans pour l'amélioration de la production, l'encadrement des paysans et l'octroi des microcrédits. Une étude serait envisageable pour voir les besoins des paysans, leurs proposer les besoins en produit de consommation et acheter chez le paysan au lieu de dépendre de l'extérieur comme la Zambie ou le Zimbabwe. Si le régime colonial avait réussi, c'était avec l'agriculture qui alimentait les entreprises, on peut le remarquer avec les différentes fermes qui environnaient la ville de Lubumbashi. Dans les milieux ruraux, c'est la culture du coton avec Cotongo devenu Cotolu, du tabac avec la Taba-Congo devenu TabaZaïre et d'autres cultures comme l'arachide, la canne à sucre, etc.

\section{Développement de l'agriculture au Katanga}

\section{A. Secteur agricole}

La devise dans le premier temps doit être « la consommation des produits agricoles de la RDC, par une hausse de droit de douane pour les produits agricoles importés.

La province du Katanga est favorable aux cultures industrielles et vivrières. Les cultures vivrières importantes qui y sont souvent pratiquées sont les suivantes : maïs (base principale de l'alimentation pour la grande majorité de la population), manioc, arachide, haricot, riz et patate douce.

Les cultures maraîchères sont régulièrement pratiquées aux alentours des grandes villes de l'hinterland minier (LUBUMBASHI, LIKASI, KOLWEZI et environs). Elles constituent une principale source de revenus pour de nombreux ménages

La politique du gouvernement provincial a permis l'exploitation de cultures vivrières telles le maïs, le manioc, le haricot, la patate douce. Ces produits connaissent une forte tendance à la baisse du prix sur le marché. Mais les besoins alimentaires de la population Katangaise avec le mouvement de la population ne peuvent aujourd'hui être couverts par la production locale du fait du faible taux de la production vivrière. L'importation des produits vivriers par la douane de Kasumbalesa vient combler cette faible production locale.

Le maïs n'est pas seulement un produit vivrier, il est aussi une culture industrielle. La culture mécanisée du maïs sur des emblavures de 5.000 ha dans les environs de la ville de LIKASI montre que le maïs sert aussi à des besoins industriels. Ce maïs était transformé en farine dans la Province pour les besoins ménagers par un certain nombre de minoteries : Minoteries de Kakontwe (MINOKA), TARIKA Frères, AMATO Frères et la Commerciale 
Industrielle et Agricole (COMICA), une partie de la production était utilisée comme matière première dans l'industrie brassicole. ${ }^{8}$

La tendance à la baisse est constatée également au niveau des cultures industrielles telles que le coton, le tabac, le palmier à huile, la canne à sucre, la banane, l'ananas, l'orange ainsi qu' à la fermeture des industries textiles et agroalimentaire.

La culture du coton, du tabac et du palmier à huile était considérée comme la principale source de revenu pour des paysans. Ces cultures alimentaient les unités agro-industrielles à savoir SOLBENA, TABAZAÏRE, FILTISAF et COTONCO (COTOLU).

\section{B. Élevage}

Dans le domaine de l'élevage la province dispose des potentialités importantes pour le développement de ce secteur. L'espace pour la zone d'élevage représente $10 \%$, ce qui peut donner plus ou moins 5.000.000 d'hectares dont 1.850.000 sont des pâturages naturels. ${ }^{9} \mathrm{La}$ province du Katanga dispose des vastes étendues des savanes pâturables, un sol et une flore agropastorales très riches qui offrent des possibilités favorables à l'élevage des bovins, ovins, porcins et volailles. Jadis, l'élevage des bovins de ranching représentait $75 \%$ que comptait le pays (ELGYMA, GRELKA, KIPIRI, LYAMPENDA, KUNDELUNGU, ONDE...).

L'élevage pratiqué dans les milieux ruraux est un élevage de prestige. La viande est consommée rarement. L'élevage des bovins est destiné à résoudre les différents problèmes que connaissent les paysans (dot, scolarisation des enfants, etc.).

\section{La pèche et la pisciculture}

Dans domaine de la pèche, le fleuve Congo, ses affluents et plusieurs lacs sont très poissonneux offrent des potentialités de pratiquer la pèche industrielle, ce qui peut résoudre le problème d'alimentation en poisson que connaît la province du Katanga. Le constat fait état pratique la pêche artisanale par la population rurale : au filet, à la nasse et parfois même un produit toxique naturelle qui est versé dans la rivière. La technique d'intoxication des poissons est à interdire, car elle extermine même les alevins. La population n'a pas la culture du respect de la réglementation en matière de la pêche. Plusieurs fois les filets ont été brulés pour décourager la clandestine.

La province dispose des principales rivières où la pèche peut être pratiquée, c'est notamment : la Lufira, la Luapula, la Luvua, la Lubilanshi, la Lomami, la Lukuga et la Lufiko.

En dehors des rivières nous avons aussi les lacs Tanganyika, Moero, le Banguelo, Kisale, Upemba et la suite des lacs compris entre Bukama et Mulongo, ainsi que les lacs de

8 Plan triennal du Katanga, p. 3.

9 Plan triennal du Katanga, p. 3. 
retenue de Nzilo et de Lufira. La pêche semi-industrielle est pratiquée à Kalemie, Kilwa et Kasenga, mais actuellement cette pèche connaît une baisse d'activité.

La pisciculture commence à être pratiquée de plus en plus dans la ville de Lubumbashi. La pisciculture est une activité à généraliser dans toute la province pour pallier au problème de la pèche pendant la période de fermeture.

\section{La commercialisation des produits}

Autrefois, l'agriculteur produisait d'abord pour consommer. La finalité actuellement est la mise sur le marché de la production. Comme toute entreprise, l'exploitation doit se procurer sur un marché en amont. Ce marché est étudié pour savoir quelle type de production. Mais la production au Katanga du type familiale se procure sur le marché en aval parce que les familles paysannes doivent faire face aux mêmes besoins que ceux qui habitent les villes (soins médicaux, logement, scolarisation des enfants, nourritures, habillement, etc.)

La province de Katanga dispose d'un réseau routier qui part de Kasumbalesa-Lubumbashi, Likasi, Kolwezi, Dilolo, Sandoa, Kapanga, Kamina, Kasenga, Pweto, Nyunzu, Kabalo, Bukama, Manono, Kongolo, Kilwa et Kalemie. Ce réseau est constitué de $5.600 \mathrm{Kms}$ des routes d'intérêt général et $13.000 \mathrm{Kms}$ des routes de dessertes agricoles. Le réseau routier de la province se trouve dans un état de délabrement, exige un financement important pour sa réhabilitation. Cependant les efforts visibles pour la réhabilitation des routes principales sont louables.

Le réseau ferroviaire est capable de relancer l'agriculture et permettre l'évacuation des produits agricoles. C'est la Société nationale des chemins de fer du Congo, SNCC en sigle qui exploite ce réseau qui relie les différents centres : Sakania, Lubumbashi, Likasi, Kolwezi, Dilolo, Kamina, Kalemie, Bukama, Kabongo, Kaniama et Kongolo. Cette entreprise connaît d'énormes difficultés qui sont lié entre autre à la chute de la Générale des carrières et des mines (Gécamines).La réhabilitation de la SNCC devra passer par l'acquisition des locomotives, des wagons et aussi de l'aménagement de la voie.

\section{E. L'agriculture industrielle au Katanga}

La politique du gouvernement provincial du Katanga consistait à demander aux entreprises d'exploitation minière d'investir dans l'agriculture et le social des populations. La contraction des activités minières des entreprises qui œuvrent au Katanga a depuis deux ans contraint un bon nombre de ces entreprises à se tourner vers la production agricole en faisant usage de la mécanisation. Dans la Province du Katanga spécialement des opérateurs du secteur agricole de l'hinterland minier recourent à l'usage des tracteurs pour augmenter les superficies cultivées et améliorer leur productivité. Ces opérateurs se retrouvent autour des villes suivantes : Lubumbashi, Likasi, Kambove, Fungurume, Kolwezi et Sakania. Cette politique agricole vise à créer la disponibilité de la nourriture en quantité suffisante, principalement le maïs. Or l'autosuffisance alimentaire vise les trois composantes qui sont la dis- 
ponibilité, l'accessibilité et l'utilisation. Bien que la disponibilité soit le cheval de bataille du gouvernement provincial, l'accessibilité et l'utilisation de la nourriture posent encore des sérieux problèmes suite aux revenus de nombreux ménages.

Cette politique provinciale a permis aux entreprises minières d'acquérir des espaces pour la production agricole. Ils ont recruté des agronomes et des travailleurs pour répondre à cet appel du gouvernement provincial au détriment de la population paysanne qui a continué avec l'agriculture artisanale. Les mérites de cette politique est la stabilisation du prix de maïs sur le marché. Le gouvernement provincial s'est félicité de ces efforts qui sont de réduire la pauvreté et d'atteindre l'autosuffisance alimentaire. A titre illustratif, l'entreprise BAZANO dans la ville de Likasi pour répondre à l'appel du gouvernement provincial a acquis deux moissonneuses-décortiqueuses. Ces machines pèsent les sacs de $50 \mathrm{kgs}$ et entament le processus de mise en sac. ${ }^{10}$

Les entreprises au Katanga ont consacré d'énormes moyens pour leur implication massive dans le développement de l'agriculture. Cette politique est loin de la réduction de la pauvreté pour répondre aux Objectifs du Millénaire pour le Développement (OMD) que le pays s'est fixé de réduire de moitié, à l'horizon 2015 : le nombre de sous-alimentés et de celui des plus pauvres en général. Les Objectifs Millénaire ont pour mission de permettre à chaque citoyen de faire face aux besoins élémentaires.

\section{Les potentialités du Code agricole pour le Katanga}

La politique agricole de la province du Katanga doit prendre en compte la planification, la production, l'organisation du marché, la fixation de prix, l'équipement rural et l'habitat. La loi devra tenter d'appréhender le problème agricole dans sa globalité pour essayer d'organiser une évolution souhaitée de la paysannerie. ${ }^{11}$ L'élaboration d'une politique agricole nationale par le ministre de l'agriculture qui sera adopté en conseil des ministres et qui devra être sanctionnée par un Décret du Premier ministre devra tenir compte de la spécificité de chaque province. Le gouvernement est tenu de présenter un rapport sur l'exécution du plan de production agricole et de commercialisation des produits agricoles chaque année avant la première session de l'année civile (Articles 7, 8 et 9);

Chaque province élabore un plan de production agricole par le biais de son ministre provincial de l'agriculture après avis du conseil rural provincial de gestion. Ce plan de production est sanctionné par un arrêté du gouverneur après l'approbation par le gouvernement provincial (Article 10). Le plan de production agricole permet d'intégrer les trois formes d'exploitation pour la relance de l'agriculture dans la province. L'expérience de l'époque coloniale qui a continué vers les années 70 avant la démocratie peut être mise à profit pour le développement de l'agriculture. L'obligation sera faite à chaque agriculteur de produire

10 «Ferme BAZANO, La récolte du maïs..., une joie partagée », in Renaître, n06/07, Juin-Juillet 2010, XVIII e année, p. 67.

11 Jean Tournier, Les bases économiques et humaines de l'activité agricole, Op.cit, p. 320. 
pour la consommation et la commercialisation. La province affecterait un agronome superviseur par secteur ou chefferie qui exigera la variété des cultures après une étude du sol pour répondre à la politique du gouvernement. En dehors des aliments de base (maïs, manioc, haricot, légumes, patates douces) d'autre cultures commerciaux doivent être envisagées (coton, canne à sucre, huile de palme, arachides, orange, citron, bananes, ananas, riz, pomme de terre, tourne sol, etc.). L'État par un partenariat local, régional ou international peut leur trouver les acheteurs.

Un arrêté du ministre de l'agriculture devra déterminer les modalités d'organisation et de fonctionnement du cadastre agricole tant au niveau national que provincial, ce service créé par le code agricole a pour mission d'octroyer des permis d'exploitation, d'assurer la bonne administration des terres rurales, de faire le constat de mise en valeur et de conserver des documents cartographiques (Article 12). L'avantage qu'offre le cadastre agricole serait de réduire sensiblement les conflits fonciers que le Katanga enregistre ce dernier temps.

Le code crée au niveau provincial un conseil agricole rural de gestion qui a pour but d'organiser l'encadrement du paysannat et sa structuration en coopérative, de vulgariser les textes, de sécuriser les paysans et les détenteurs des droits fonciers, sensibiliser les agriculteurs sur les prix et marchés agricoles, analyser les pistes de développement du milieu rural, analyser l'impact négatif environnemental et proposer les solutions et sécuriser les structures juridiques du paysannat et des coopératives (Article 13). La province du Katanga compte 55 chefferies et 37 secteurs qui donnent un total de 92 entités, le gouvernement devra affecter au moins un agronome par secteur et un vétérinaire doté d'un moyen de déplacement (moto) pour l'encadrement des agriculteurs paysans en vue de garantir le remboursement du crédit affecté aux semences. L'évaluation des agronomes et vétérinaire se fera au vu des résultats lors de la récolte.

Le code crée un Fonds de gestion des risques et des catastrophes agricoles doté de la personnalité juridique. Ce Fonds est alimenté par une dotation budgétaire pour les interventions dans les secteurs agricole en cas des calamités naturelles et des épidémies. Un arrêté du ministre devra fixer l'organisation et le fonctionnement (Article 59);

Une autre possibilité que le code offre est la création de la commission chargée de la gestion et du contrôle des infrastructures agricoles au niveau de chaque province. Un arrêté du gouverneur devra fixer son organisation et son fonctionnement. Une allocation budgétaire est prévue par le gouvernement provincial pour l'entretien et la maintenance des infrastructures de base (voies de dessertes agricoles et paiement cantonnage manuel) (Articles 87 et 88 );

Pour une formation initiale et continue en faveur des agriculteurs, cette formation est soutenue par l'État par les fermes écoles. Un arrêté du ministre fixe les modalités d'organisation et de fonctionnement. Cette formation est nécessaire pour les paysans, les exploitant agricoles sont sensé avoir une unité, un capital et un personnel qualifié pour leur exploitation car ceux-ci visent les bénéfices, il s'agit d'une activité économique (Article 95).

La recherche scientifique en matière agricole a pour but d'apporter des réponses appropriées, durables et respectueuses de l'environnement. Cette recherche est organisée par les 
institutions publiques et privées nationales. Ces institutions constituent le Système National de Recherche Agricole. Un arrêté du ministre fixe l'organisation et le fonctionnement (Articles 98 et 100).

\section{L'importance de la terre}

La terre est depuis toujours la base de la production agricole, donc de l'alimentation des hommes. Elle a constitué la richesse par excellence, la source de toutes autres richesses. ${ }^{12}$ Chez beaucoup des congolais, le désir de possession de la terre est très vif parce qu'il constitue un motif de dignité sociale (aspect psychologique). La possession de la terre accorde au possesseur une identité (aspect politique). La possession de la terre est une source de revenu, un capital (aspect économique).

Mais une politique devra permettre au propriétaire de fond de le mettre en valeur sous peine d'être concédées à un exploitant qui en fait la demande. Le constat fait des terres environnantes de la ville de Lubumbashi, ces terres sont occupées par les riches qui ont érigé des grandes fermes à l'exemple de la Ferme Nazeme, Hyper-Psaro, Ferme Naviundu, Ferme Kasapa, Ferme Kisanga, Ferme Mimbulu, Ferme Kilobelobe, Parc Muyambo, Ferme Espoir, Ferme Futuka, etc. Les paysans des communes annexes qui entourent la ville de Lubumbashi n'arrivent pas à trouver les terres pour des activités agricoles.

L'article 52 reconnaît à chaque communauté locale les droits fonciers coutumiers exercés collectivement ou individuellement conformément aux articles 388 et 389 de la loi $n$ ๑73/021 du 20 juillet 1973 portant régime général des biens. Une contradiction se dégage à la limitation à 3 hectares et un hectare et demi si l'on veut développer l'agriculture paysanne. Il est difficile à ces jours de trouver des terres disponibles dans les chefferies, secteurs ou groupements. Ces terres font l'objet d'une l'appropriation individuelle des membres du groupe. Elles comprennent des réserves des terres de cultures, de jachère, de pâturage et de parcours, et les boisements utilisés régulièrement par la communauté locale.

Un acteur nouveau, l'appropriation de terres agricoles sur les communautés locales est soumise aux règles de la prescription coutumières. (Art 56) Quid d'un industriel qui s'arrange avec un chef coutumier pour exploiter les terres des communautés locales, il y a le risque de fraude au fisc. Quel est la sécurité juridique en cas de conflit peut-il produire l'acte écrit comme preuve de la propriété devant les cours et tribunaux.

Le code agricole se substitue au droit de la famille, principalement les successions. L'exploitation agricole de type familiale ou familiale en cas de succession l'exploitation passe entre les mains d'un seul héritier qui doit à son tour compenser cette acquisition aux autres héritiers par la prise en charge ou d'autres mécanismes (Article 57). 


\section{Mutualité sociale agricole}

La mutualité sociale agricole regroupe les caisses d'assurances sociales agricolLe regroupement de village (la solidarité africaine est parfois remise en cause par la multiplicité des villages qui bloque l'action de développement). Les tracteurs qu'on montre à la télévision ne doivent pas être des objets des campagnes mais plutôt des outils de production. L'exploitation du sol sans repos, sans fertilisant rend la production faible malgré des vastes étendus que les agriculteurs exploitent. Le souci de rendement fait exploiter les gens de vastes superficies avec une utilisation abusive ou tardives des fertilisant. Il est aujourd'hui un fait vrai les gens parcourent des grandes distances pour cultiver, ils sont parfois obligés de résider aux champs pendant les saisons culturales.

Le code agricole crée un fonds pour la promotion de la recherche agricole, ce fonds est alimenté par les redevances provenant de la rémunération des résultats de la recherche par les utilisateurs (Article 105). Ces recherches peuvent l'amélioration des espèces agricoles. En plus du Fonds de la recherche, le code prévoit la création de la Caisse National de crédit agricole et le Fonds Spécial de crédit Agricole. La Caisse octroie les crédits aux exploitants industriels et de type familial (Articles 108 et 110). L'inquiétude repose sur la multiplicité des caisses de crédits au lieu d'un service qui s'occuperait d'encourager l'exploitation agricole par les crédits. Ce service se trouve au niveau national comme une coordination mais les fonds sont gérés par les provinces qui ont des succursales dans les districts et territoires.

Il est institué un Observatoire du secteur agricole dénommé « Observatoire », il aura des représentations en provinces. Un décret présidentiel fixera les statuts, l'organisation et le fonctionnement (Article 151).

\section{Les problèmes fonciers}

La propriété foncière est la propriété des biens-fonds, c'est-à-dire des terres et des maisons. Elle a une grande importance en agriculture, la terre étant la base indispensable de l'activité agricole. $^{13}$

Le code agricole parle de trois types d'exploitation : l'exploitation industrielle, l'exploitation familiale et l'exploitation du type familial. L'acquisition des terres agricoles peuvent être situées soit dans une circonscription urbaine soit dans une circonscription rurale. Ces terres sont destinées à l'agriculture ou l'élevage.

Les articles 65 à 71 du code créent une structure de résolution de conflit, ce qui crée un chevauchement avec le droit coutumier qui tranche de nombreux litiges des terres par le biais des chefs de collectivités, secteurs ou groupements. Le code fait du comité foncier, un tribunal de litige en matière de terres rurales.

La concession agricole peut être octroyée à tout exploitant industriel qui en fait la demande conformément aux articles 80 et suivants et 110 et suivant de la loi n ${ }^{\circ} 73 / 021$ du 20 
juillet 1973 portant régime général des biens, régime foncier et immobilier et régime des sûretés.

Cette concession peut être soit une concession perpétuelle soit une concession emphytéotique. Les terres agricoles en exploitation et en utilisation peuvent l'être par des industriels qui sont les personnes physiques ou morales de droit privé ou de droit public.

L'octroi de toute concession agricole sur les terres rurales est subordonné à l'enquête préalable telle que prévue par les articles 193 à 203 de la loi nº73-021 du 20 juillet 1973 portant régime général des biens, régime foncier et immobilier et régime des sûretés.

La même loi en ses articles 387, 388 et 389 prévoit Les terres occupées par les communautés locales devenues des terres domaniales, à partir de l'entrée en vigueur de la loi $\mathrm{n}^{\circ}$ 73-021 du 20 juillet 1973.

Les terres occupées par les communautés locales sont celles que ces communautés habitent, cultivent ou exploitent d'une manière quelconque individuelle ou collective conformément aux coutumes et usages locaux. Les droits de jouissance régulièrement acquis sur ces terres seront réglés par une ordonnance du président de la République.

Les conflits de terres dans les milieux ruraux sont très récurrents. Ce code pour palier à cette situation a crée un cadre non juridictionnel décentralisé appelé Commission Foncière Locale Permanente. Cette Commission est composée des acteurs fonciers étatiques d'un côté et non étatiques de l'autre. La mission que le code à confier à cette commission est de réguler les opérations foncières.

L'objectif global est d'assurer aux acteurs ruraux, dans leur diversité, l'accès équitable au foncier, la garantie, la sécurité et la jouissance de leurs investissements, la gestion efficace des différends fonciers afin de contribuer à la réduction de la pauvreté, à la consolidation de la paix sociale et à la réalisation d'un développement durable qui sauvegarde l'environnement.

Il s'agit de garantir le droit d'accès légitime de l'ensemble des acteurs ruraux au foncier, dans une dynamique de développement rural durable, de lutte contre la pauvreté et de promotion de l'équité;

L'objectif est de contribuer à la viabilité et au développement des collectivités territoriales, par la mise à leur disposition des ressources foncières et d'outils de gestion foncière efficace et de prévenir et réduire les litiges liés au foncier. Les conflits fonciers entre les membres de la communauté locale ne sont pas recevables devant les instances judiciaires s'ils n'ont été préalablement soumis à la procédure de conciliation, à l'initiative de l'une des parties devant le comité foncier.

Cette procédure interrompt le délai de prescription prévu en droit commun, dès la réception de la demande de conciliation par le comité foncier, sous réserve toutefois que la demande devant la juridiction compétente en cas de non conciliation, soit introduite dans le délai maximum de trois mois à compter de la réception du procès verbal de non conciliation par la partie diligente.

Lorsque le comité foncier est saisi d'un conflit foncier, il adresse une invitation à comparaître en séance de conciliation dans les quinze jours. En aucun cas l'invitation ne peut 
obliger l'une des parties à se présenter dans un délai inférieur à quinze jours. Le comité foncier procède à un échange de vue sur l'objet du litige et vérifie si les parties sont disposées à se concilier. A la fin des échanges des vues, le comité foncier établit un procès verbal constatant la conciliation ou la non conciliation.

Le procès verbal est signé par les membres du comité foncier et les parties. Celles-ci en reçoivent copies. Si à la troisième invitation dûment reçue, une partie ne se présente pas, le comité foncier établit un Procès Verbal de carence valant constat de non conciliation. En cas de conciliation, la partie la plus diligente fait apposer la formule exécutoire sur le procès verbal auprès du président du tribunal de paix compétent. Si un des membres du comité foncier a des liens familiaux, voire d'amitié avec l'une des parties en conflit, ce dernier est tenu de se déporter. S'il ne le fait pas, l'autre partie peut le récuser et dans ce cas, il motive les raisons de cette récusation. ${ }^{14}$ Cette procédure est possible lorsqu'il s'agit des conflits entre membres de la communauté locale. Mais lorsqu'il s'agit d'un conflit entre un paysan et un industriel, la loi foncière protège le tenant des titres fonciers d'où la nécessité de la sécurisation des communautés locales.

\section{Les assurances}

La création d'un fonds de gestion des risques et des catastrophes agricoles doté d'une personnalité juridique, il est alimenté par une dotation budgétaire (Article 59). Or, en connaissant les réalités congolaises ce fonds n'a pas de chance d'exister.

L'article 62, toutes maladies cryptogamiques ou parasitaires doivent être signalées à un vétérinaire ou un agronome les plus proches qui ne sont pas encore installés. Le fonds est localisé à Kinshasa, l'expérience montre la lenteur administrative, la victime de la catastrophe risque d'être indemnisée même 10 ans après. Ce rôle serait joué par les assurances avec une branche spéciales agricoles.

En lisant attentivement le code agricole, il y a encore beaucoup d'étapes à franchir, c'est la mise sur pied de différentes structures pour son effectivité qui risque de prendre encore longtemps si ne pas rester un texte sans objectif comme la plus part des textes congolais.

\section{CONCLUSION}

\section{A. Critique du code agricole}

Le premier problème que le code agricole présente est celui de n'avoir pas associé les autres acteurs importants (secteur minier, les finances, affaires foncières, les gouverneurs et les paysans).

14 Articles 66 à 71 du Code agricole. 
Le cadastre agricole vient se greffer aux affaires foncières, au cadastre minier et forestier qui créerait des conflits. Au lieu d'éclater les affaires foncières en secteur agricole, minier et forestier, on gardera l'unique cadastre comme la Douane et la contribution.

Aussi, la réduction du rôle de chef de secteur et collectivité qui sont des responsabilités dans les entités décentralisées risque de bloquer le développement dans les milieux ruraux. Le code agricole accorde au juge un pouvoir exorbitant en cas de conflit, celui de trancher en faveur de l'intérêt de l'agriculture qui malheureusement n'est pas précisé.

L'article 38 parle de l'État qui détermine les terres destinées à l'usage agricole ou pastoral, que devient alors le rôle du cadastre agricole. On le sait bien que ce sont les affaires foncières.

Sur le plan administratif les relations ne sont jamais bien définies entre les divisions de l'agriculture et le ministère provincial de l'agriculture qui a en sa charge trois autres secteurs qui dépendent des ministères différents au niveau national.

La lecture faite du code agricole montre le souci d'avoir un instrument pour contribuer au budget national au lieu de l'amélioration des conditions de vie des populations rurales. L'article 48 limite l'étendue de l'exploitation agricole à 3 hectares pour l'exploitation du type familiale et à un hectare et demi pour l'exploitation familiale.

\section{B. Les perspectives de relance agricole}

La première démarche c'est de consommer les produits locaux, en amenant le producteur à produire en fonction des besoins du marché local. Le paysan est le cœur de la nation. Si leurs produits arrivent au centre de consommation le prix serait réduit de 10 fois moins.

La deuxième démarche peut être la mécanisation de l'agriculture paysanne, la disponibilité du marché pour les semences, engrais, pesticides et autres accessoires pour l'agriculture. Ces tracteurs de grande capacité peuvent être mis à la disposition des paysans en raison d'un tracteur par secteur ou collectivité. A la période de la récolte le total des dépenses peut être défalqué sur la production obtenue. Ce fonds peut constituer un fonds de roulement et payer un deuxième tracteur.

Une autre possibilité c'est celle des exploitants des entreprises agricoles privées, ils peuvent aider l'État dans l' achat des produits pour les paysans en tenant compte du prix réel sur le marche, leurs encadreurs étant au service du gouvernement peuvent aider les paysans à augmenter leur capacité de production et sachant que eux produisent pour un marché sûr. La mécanisation aura un impact sur l'environnement. Il s'agira de demander la réunification des villages et la technique de réunification des champs pour former des grands champs. Cet esprit peut amener les paysans à la création de coopérative en cas refus les réunir et délimiter l'espace de chacun. Cette réunification des villages va entrainer la création des œuvres communes (les terrains de jeux, les hôpitaux, marché, les écoles, etc.). Au bout de cinq ans minimum et maximum dix ans interdiction formelle de voir une maison en taille, les villages réunifiés doivent être à coté d'une rivière pour accélérer la fourniture en eaux potable en vue d'éviter des maladies d'origine hydrique. 
Avant d'envisager l'agriculture basée sur les exportations, il faut d'abord s'assurer que les petits agriculteurs et les communautés rurales sont assistés pour réduire les inégalités entre la ville et le village. Il y a moyen d'encadrer leur production pour le faire contribuer à la production nationale. L'objectif du code agricole est d'atteindre l'autosuffisance alimentaire (hydrates de carbone et protéine), d'éradiquer la pauvreté et la promotion de l'équilibre entre les provinces. Il s'agit d'une politique du gouvernement qui nécessite un partenariat entre le gouvernement et les privés étant donné que l'État cherche le développement et les privés cherchent le profit. L'agriculture d'exportation est une matière première pour l'industrie agro-alimentaire et sera considéré de la même manière que l'exploitation minière, encore actuellement base de l'économie de la RDC.

Les universités doivent être considérées comme les laboratoires. Les étudiants de médecine vétérinaire et ceux des sciences agronomiques peuvent être affectés en stages obligatoires dans les fermes et champs de paysans sous la supervision des agronomes et vétérinaire de collectivité.

Le code agricole est un document conçu pour attirer le financement des capitaux au sein de la communauté internationale et des banques africaines pas un instrument d'impulsion pour le développement des milieux ruraux. La preuve est la place accordée au paysan dans le code. La politique agricole en République Démocratique du Congo en général et au Katanga en particulier doit identifier les besoins réels des populations locales pour permettre leur implication et l'appropriation de différents projets de relance de l'agriculture. L'impact sera l'amélioration de la qualité de vie de la population et de penser aux conditions de fixation des populations dans les centres à haute productivité dans le domaine de la pêche, de l'élevage et de l'agriculture pour soutenir le développement des milieux ruraux et la réduction de la pauvreté.

Le Code agricole est un texte très ambitieux sans pragmatisme qui a moins de chance d'atteindre les objectifs. La culture de commission est loin d'être guérie chez le congolais qui aime de commission pour être toujours considéré comme expert. Il est raisonnable de penser à des structure pérennante où les gens ont de l'emploi et ont un salaire à titre de rémunération de leur travail. L'article 109 organise la caisse nationale de crédit agricole chargée de coordonner et de contrôler les opérations de crédit agricole. Cette caisse est à Kinshasa et elle consent aux caisses établies en province des avances qui sont utilisées en prêts à court, à moyen et à long terme. L'octroi de crédit passe par l'avis d'une commission provinciale de crédit agricole nommée par le gouverneur. Elle est constituée des membres qui proviennent du secteur public (fonctionnaire de l'État) et les privés intéressés à l'exploitation agricole. La commission d'experts vérifie autant que possible les indications données par le demandeur du crédit et fait rapport au président, ce qui est la tache courante de la commission. Mais, il apparaît les indemnités qu'il convient à payer à la commission au terme de leur expertise sur l'étude du dossier. Ces indemnités sont indexées sur le crédit, c'est le demandeur qui paie les experts ou les membres de la commission qui ont pour tache d'étudier les dossiers de demandeurs en vue de leur octroyer du crédit. La logique serait que les membres de la caisse de crédit agricole soient des permanents payés pour leur tra- 
vail au lieu de décourager le demandeur du crédit si l'on tient à la relance de l'exploitation agricole.

Les différents services créés par le code sont des services inexistants et pour leur opérationnalité doivent obtenir soit le décret présidentiel, soit l'arrêté du ministre ou du gouverneur. La démarche devient longue au risque de bloquer le développement du secteur agricole. A notre avis certains services à créer pouvaient dépendre des autres services qui existent, d'autres qui sont tombés en faillite qui nécessite une étude minutieuse pour appuyer le secteur agricole à titre illustratif la banque de crédit agricole. Le cadastre agricole demande l'éclatement des affaires foncières, le fonds de gestion des risques et catastrophes, il existe les assurances en République Démocratique du Congo, l'INRA dans le cadre de la recherche avec les différentes institutions, la commission chargée de la gestion et du contrôle des infrastructures agricoles au niveau de chaque province, les équipes existent en milieu rural, ils ont besoin d'une rémunération pour la protection des infrastructures.

Il est malheureux de voir certains riches qui dépossèdent les villageois de leur terres pour y installer les fermes, les parcs, les cites touristiques au tour de grands centres, qui utilisent des tracteurs dans leur champ qui voient à côté de leur fermes de villageois qui font leur champ par des méthodes traditionnelles. L'importation des tracteurs ne peut jamais développer l'agriculture ou réduire la pauvreté. Il faut que la population s'approprie l'activité agricole au lieu de décider à leur place. Pour cela, il serait souhaitable de créer les sociétés d'aménagement et d'établissement rural qui auront pour tâche d'aménagement et de création d'exploitation agricoles viables. Ces entreprises peuvent acheter ou louer les terres à vocation agricole, créé par regroupement des parcelles pour le fermage. Elles peuvent réaliser les travaux pour les paysans qui en font la demande.

Le discours de la relance agricole est devenu un slogan, une campagne politique. Le taux de chômage décrié dans le monde ne touche en rien le paysan qui ne pense qu'à son champ, qui doit vendre ses produits de champs pour faire face aux dépenses de la vie nécessaire. Mais il est comptabilisé dans le PIB. Le colonisateur pour aider villageois avait pensé à l'industrie textile, tous les paysans cultivaient le coton et savaient le moment du marché pour avoir un peu d'argent. On a vu à cette même époque les fermes autour des villes pour nourrir la population de la ville. Aujourd'hui c'est le contraire.

La réhabilitation des routes par le cantonnage manuel en attendant les grands travaux à long terme peut résoudre la question d'évacuation. L'impact serait que le cantonnier s'occuperait de son travail avec un salaire qui lui permettra d'acheter et faire le cantonnage et ensuite faire le champ pour survivre parce que la rémunération ne lui permet pas de vivre sans une autre activité. 


\section{BIBLIOGRAGHIE}

1. Textes de lois

- Code agricole

- Loi n73-021 du 20 juillet 1973 portant régime général des biens, régime foncier et immobilier et régime des sûretés.

\section{Ouvrages}

- Azoulay, G. et Dillon, J-C., La sécurité alimentaire en Afrique, Paris, Ed. Karthala, 1993.

- Dibwe dia Mwembu, Histoire des conditions de vie des travailleurs de l'Union Minière du Haut-Katanga/GECAMINES (1910-1999), Lubumbashi, PUL, 2001.

- Tulkens et M. Van de Kerchove, Introduction au droit pénal, aspects juridiques et criminologiques, $3^{\mathrm{e}}$ éd, Bruxelles, Story-Scientia, 1997.

- Fierens, J., Droit et pauvreté, droits de l'homme, sécurité sociale, aide alimentaire, Bruxelles, Bruylant, 1992.

- Jean Tournier, Les bases économiques et humaines de l'activité agricole, $3^{\mathrm{e}}$ édition, Paris, Lavoisier, 1989.

- Lambrechts, A. et Bernier, G., Enquête alimentaire et agricole dans les populations rurales du Haut-Katanga, Elisabethville, Ed. Cepsi, 1961.

- Mukadi Kankonde, Sécurité alimentaire au Congo-Kinshasa : production, consommation et survie, Paris, L'Harmattan, 2001.

- Mukendi Nkashama et Kabeya Mukamba, Guerres et mutations sociolinguistiques en République Démocratique du Congo (1960-1999), PUL, Tome II, Lubumbashi, 2000.

- Muyer Oyong, Impératif du développement et réforme de l'administration locale au Zaïre, Kinshasa, P.U.Z., 1986.

- Nkuku Khonde, C. et Rémon, M., Stratégie de survie à Lubumbashi (R-D Congo), Paris, L'Harmattan, 2006.

- Pisani, E., Pour l'Afrique, Paris, Ed. Odile Jacob, 1988, p. 17.

\section{ARTICLES ET REVUES}

- FAO, Colloque sur la sécurité alimentaire en République Démocratique du Congo, Kinshasa, Alliance Belgo Congolaise, Rapport Final, 2003.

- FAO/RDC, Mission à moyen terme de la FAO : "les grands enjeux pour la RDC en matière de production alimentaire », Table ronde sur l'agriculture, Kinshasa, 19-20 mars, 2004.

- Fédération internationale des sociétés de la Croix-Rouge et de la Croissant-Rouge, Comment évaluer la sécurité alimentaire, Guide pratique des sociétés nationales africaines, Myanmar, 2004. 
- Fleury, A., Construire la ville avec l'agriculture au Nord et au Sud, Colloque, Bruxelles, Septembre 2002.

- Mafulu Uyind'a Kanga, La place du maïs dans l'alimentation de la population ouvrière noire de l'Union Minière du Haut Katanga, Mémoire d'Études approfondies, Facultés de Lettres, Université de Lubumbashi, 2011

- Mertens Ngoy Limbu Mbayo, «Le travail législatif en agriculture urbaine, réflexion à partir de la ville de Lubumbashi », in villes du Nord et villes du Sud à la rencontre de l'agriculture, Bruxelles, Septembre 2002.

- OCHA 2005, République Démocratique du Congo : analyse de la sécurité alimentaire et de la vulnérabilité, Kinshasa, SENAC, 2005.

- Renaître, Spécial 30 juin, Mensuel chrétien d'information et d'opinion, XVIII e année, n ${ }^{\circ}$ 06/07, Juin-Juillet 2010.

- Tshingombe Muluday, F., « La RDC face à la crise alimentaire mondiale », in CongoAfrique, $\mathrm{n}^{\circ}$ 429, novembre 2008, pp. 737-746.

- Victor Dibualonji, "L'agriculture intra et péri-urbaine à Lubumbashi (RDC) », in villes du Nord et villes du Sud à la rencontre de l'agriculture, Bruxelles, Septembre 2002. 